\title{
Finite Temperature Effects in One-dimensional Mott-Hubbard Insulator: Angle-Resolved Photoemission Study of $\mathrm{Na}_{0.96} \mathrm{~V}_{2} \mathrm{O}_{5}$
}

\author{
K. Kobayashi, T. Mizokawa, A. Fujimori \\ Department of Physics, University of Tokyo, Hongo 7-3-1, Bunkyo-ku, Tokyo, 113-0033, Japan \\ M. Isobe, Y. Ueda \\ Materials Design and Characterization Laboratory, Institute for Solid State Physics, University of Tokyo, Roppongi 7-22-1, \\ Minato-ku, Tokyo, 106-8666, Japan \\ T. Tohyama and S. Maekawa \\ Institute for Materials Research, Tohoku University, Katahira 2-1-1, Aoba-ku, Sendai, 980-8577, Japan
}

(Phys. Rev. Lett., 82, 803 (1999))

\begin{abstract}
We have made an angle-resolved photoemission study of a one-dimensional (1D) Mott-Hubbard insulator $\mathrm{Na}_{0.96} \mathrm{~V}_{2} \mathrm{O}_{5}$ and found that the spectra of the $\mathrm{V} 3 d$ lower Hubbard band are strongly dependent on the temperature. We have calculated the one-particle spectral function of the onedimensional $t$ - $J$ model at finite temperatures by exact diagonalization and compared them with the experimental results. Good overall agreement is obtained between experiment and theory. The strong finite temperature effects are discussed in terms of the existence of the "Fermi surface" of the spinon band.
\end{abstract}

PACS numbers: 71.27.+a, 79.60.Bm, 71.10.Pm, 75.20.Ck

The most striking and non-trivial theoretical prediction for 1D strongly correlated systems is the spin-charge separation [1]: the degrees of freedom of an electron are decoupled into elementary excitations of spin and charge called "spinon" and "holon", respectively. Angleresolved photoemission spectroscopy (ARPES) is a powerful technique to study the spin-charge separation: Kim et al. [2] performed a pioneering ARPES work on the 1D charge-transfer insulator $\mathrm{SrCuO}_{2}$ and found that the spectra agree well with the theoretical one-particle spectra of the 1D $t$ - $J$ model with realistic parameters, identifying such spinon and holon excitations. We subsequently investigated whether the same scenario is applicable for a 1D Mott-Hubbard type insulator $\mathrm{NaV}_{2} \mathrm{O}_{5}$ [3].

Strongly-correlated systems are often characterized by the presence of a characteristic low energy scale in spite of the large energy scales of bare interaction strengths. For the Hubbard model, the relevant low energy scale is set by the superexchange interaction $J \sim 4 t^{2} / U(\ll t, U)$ rather than the bare interaction parameters, the transfer integral $t$ and the on-site Coulomb repulsion $U$. Since the photoemission spectrum is a projection of the initial state onto the set of final states, drastic finite-temperature effects may be expected for a temperature change of the order of such a characteristic low energy scale. Finitetemperature effects can be particularly drastic in 1D systems 佃 because of the existence of the "Fermi surface" of spinon excitations. In this Letter, we present the result of a temperature-dependent ARPES study on $\mathrm{NaV}_{2} \mathrm{O}_{5}$. While severe charging effects had previously prevented the measurements of $\mathrm{NaV}_{2} \mathrm{O}_{5}$ below $\sim 300 \mathrm{~K}$ [3], more conductive $\mathrm{Na}_{0.96} \mathrm{~V}_{2} \mathrm{O}_{5}$ [5] enabled us to obtain ARPES spectra at temperatures as low as $\sim 120 \mathrm{~K}$. Dispersive features of $\mathrm{V} 3 d$ character in the lower Hubbard band were found to be dramatically dependent on the temperature. In addition, we have made a comparison with theory and confirmed that the observed finite-temperature effect is due to strong correlation effect rather than the simple thermal broadening.

Recently $\mathrm{NaV}_{2} \mathrm{O}_{5}$ has widely aroused much interest as a quasi-1D quantum-spin system. Crystalographically, it is still controversial whether the $\mathrm{V}$ atoms in $\mathrm{VO}_{5}$ pyramids running along the $b$-axis of $\mathrm{NaV}_{2} \mathrm{O}_{5}$ is mixed-valent $\left(\mathrm{V}^{4+}: \mathrm{V}^{5+}=1: 1\right)$ [6.7] or uniform-valent $\left(\mathrm{V}^{4.5+}\right)[8$ ]. In the former case, this compound can be no doubt regarded as a half-filled chain [7], while in the latter case it is viewed as a quarter-filled ladder system [8,9]. At low temperatures around or below its spin-Peierls-like (SP) transition temperature $T_{S P} \sim 34 \mathrm{~K}[7]$, the difference between those two models is significant in terms of the charge ordering pattern. On the other hand, the magnetic susceptibility $\chi(T)$ well above $T_{S P}$ is successfully fitted to the Bonner-Fischer curve with $J \sim 560 \mathrm{~K}[$ ], indicating that $\mathrm{NaV}_{2} \mathrm{O}_{5}$ behaves as a good 1D antiferromagnetic Heisenberg chain in this temperature region. In fact, it is theoretically supported that it can be mapped on to the 1D Heisenberg chain [8]. Except that the SP transition is suppressed [5], the Na-deficient $\mathrm{Na}_{0.96} \mathrm{~V}_{2} \mathrm{O}_{5}$ has almost the same magnetic properties as $\mathrm{NaV}_{2} \mathrm{O}_{5}$ [10]. Though remaining an insulator, $\mathrm{Na}_{0.96} \mathrm{~V}_{2} \mathrm{O}_{5}$ is more conductive than $\mathrm{NaV}_{2} \mathrm{O}_{5}$ due to the doped holes.

Single crystals of $\mathrm{Na}_{0.96} \mathrm{~V}_{2} \mathrm{O}_{5}$ were prepared as reported in Ref. 11. They could be easily cleaved parallel to the $a b$ plane. The ARPES measurements were made using the He I resonance line $(h \nu=21.2 \mathrm{eV})$ and a hemi-spherical analyzer with angular resolution $\pm 1^{\circ}$ and 
energy resolution $80-100 \mathrm{meV}$. The measurement temperature ranged from $T=120 \mathrm{~K}(=0.21 J)$ to room temperature $300 \mathrm{~K}(=0.54 J)$. The measurements were performed for several in situ cleaves, for which we carefully cycled the temperatures of cleavage and measurements in order to exclude any extrinsic effects such as surface degradation and contamination.

Before discussing the $\mathrm{V} 3 d$ band features of our main interest, we would like to mention that the $\mathrm{O} 2 p$ band structure was found very anisotropic: ARPES spectra with momentum parallel to the $b$-axis $(k \| b)$ show rich dispersive features while those with momentum parallel to the $a$-axis $(k \perp b)$ have no dispersions, supporting the one-dimensionality of this compound. These results well agree with those of stoichiometric $\mathrm{NaV}_{2} \mathrm{O}_{5}[3]$, indicating that the $\mathrm{Na}$ deficiency has no appreciable influence on the $\mathrm{O} 2 p$ band structure. Furthermore, the $\mathrm{O} 2 p$ spectra have no obvious temperature-dependent changes, making a clear contrast to the remarkable changes of the $\mathrm{V} 3 d$ band described below.

Figures 1 (a) and (b) show the geometry of the measurements. By scanning the momentum in the Brillouin zone (BZ) as indicated, we obtained ARPES spectra along the $k \perp b$ and " $k \| b$ " cuts as shown in Figs. 1 (c) and (d), respectively. Hereafter, $k_{a}$ and $k_{b}$ are the momenta along the $a$ - and $b$ - axes in units of the inverse of the corresponding lattice constant, respectively. All the spectra have been normalized to the area of the lower Hubbard band of $\mathrm{V} 3 d$ origin between the binding energies $\left(E_{B}\right)$ of 0.0 and $2.5 \mathrm{eV}$ after subtracting the integral background. We notice that the $k \perp b$ cut [Fig. 11(c)] shows a clear temperature dependence and a weak angle dependence except for around the $\Gamma$ point $\left(\theta=\phi=0^{\circ}\right)$. In addition, the absolute intensity at the $\Gamma$ point was found to be weaker than half of those at $\phi>10^{\circ}$. These observations can be explained by the facts that the occupied V $3 d$ orbital has $x y$ symmetry lying approximately in the $a b$-plane $[12$ and therefore that normal emission from this orbital is forbidden due to selection rules [13]. We therefore conclude that the temperature dependence results from the intrinsic finite temperature effects of the $\mathrm{V} 3 d_{x y}$ band 14 and that the momentum dependence along the $k \perp b$ direction is due to the matrix element effects of the $d_{x y}$ orbital.

Figure 1 (d) shows the result for the " $k \| b$ " cut. In order to avoid the $\Gamma$ point where the matrix-element effects prohibit emission from $d_{x y}$, the $k_{a}$ value was slightly offset from the $b$-axis as shown in Fig. 1 (b). The obtained $120 \mathrm{~K}$ spectra show rich dispersing features. In going from $k_{b}=0$ to $\pi / 2$, a single peak centered at $E_{B}=0.9$ $\mathrm{eV}$ is split into two features: the splitting becomes largest at $k_{b}=\pi / 2$ with the two features located at $E_{B} \sim 0.7$ and $\sim 1.4 \mathrm{eV}$. The $0.7 \mathrm{eV}$ peak then decreases in intensity in going from $k_{b}=\pi / 2$ to $\pi$ and only a single broad peak is left at $E_{B} \sim 1.1 \mathrm{eV}$ at the BZ boundary $k_{b} \sim \pi$. The $k_{b}$-dependence of the spectra between $k_{b}=0$ and $2 \pi$ is

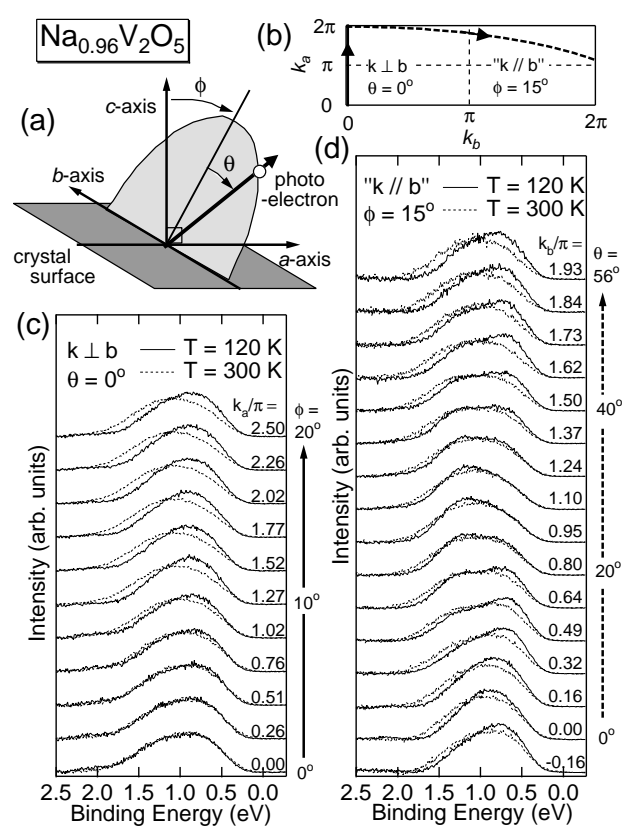

FIG. 1. (a) Definition of the take-off angles $\phi$ and $\theta$ of photoelectrons. (b) Measured cuts in the the Brillouin zone. (c) Spectra for the $k \perp b$ cut and (d) those for the " $k \| b$ " cut. Solid and dashed curves show spectra taken at $120 \mathrm{~K}$ and 300 $\mathrm{K}$, respectively. Note that each spectrum is normalized to its area and that the absolute intensities of the spectra near $\theta=\phi=0^{\circ}$ are much weaker than the others.

almost symmetric with respect to $k_{b}=\pi$, which excludes significant changes in the photoemission matrix elements between the first and second BZ's. By contrast, the $300 \mathrm{~K}$ spectra, which well agree with the previous report [3, 15], show less pronounced features than the $120 \mathrm{~K}$ spectra. The spectra at $k_{b}=0$ and $\pi$ become a broader peak with a longer tail towards high binding energies. As for the spectra at $k_{b} \sim 0.5 \pi$, the peak located at $E_{B} \sim 0.7 \mathrm{eV}$ becomes weaker and that at $\sim 1.4 \mathrm{eV}$ stronger in going from $120 \mathrm{~K}$ to $300 \mathrm{~K}$. We also performed ARPES measurements at $200 \mathrm{~K}$ and confirmed that the changes are gradual as a function of temperature. These observations are more clearly recognizable in the intensity plots (b) and (c) of Fig. 2.

By noting that the finite-temperature effects strongly depend on the momentum $\left(k \equiv k_{b}\right)$, they are clearly not due to simple thermal broadening or charging effects. The following two points may be remarked: (i) in the temperature range studied here, there is no phase transition in this compound that may give rise to such a dramatic change; (ii) the energy scale of the spectral change is not of order $k T \sim 0.03 \mathrm{eV}$ but of order $\sim 1 \mathrm{eV}$. These phenomena are obviously beyond the conventional band picture and would reflect strong correlation effects.

In order to interpret the above observations quantita- 

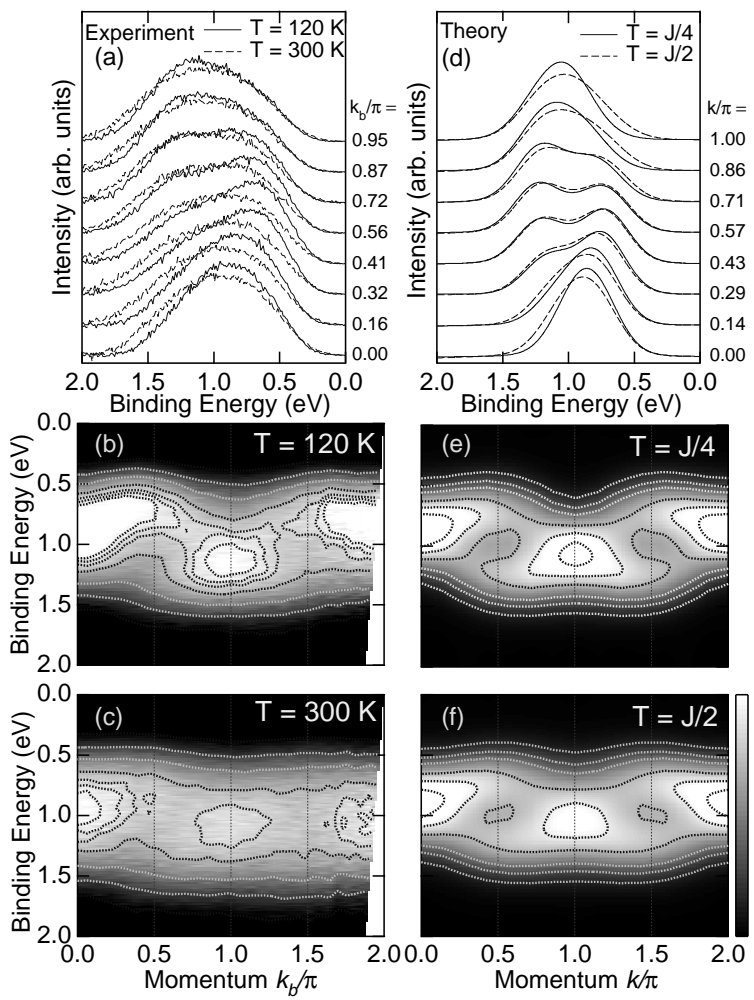

FIG. 2. Comparison between the experimental and theoretical spectra. Panel (a) shows the ARPES spectra of $\mathrm{Na}_{0.96} \mathrm{~V}_{2} \mathrm{O}_{5}$ with the intensity plots in panels (b) and (c). Panels (d), (e) and (f) show the theoretical counterparts calculated for the half-filled 14-site $t-J$ model. Here $J=0.05$ $\mathrm{eV}$.

tively, we have adopted the $1 \mathrm{D} t-J$ model to calculate the one-particle spectral function $A(k, \omega)$ at finite temperatures by the exact diagonalization method [16]. As the behavior of $\chi(T)$ well above $T_{S P}$ indicates, the $t-J$ model is valid as one of the simplest models to describe this system in the temperature region considered. It may be applicable not only to the half-filled chain case [6.,7, but also to the quarter-filled ladder case where each $d$ electron is localized in a rung of two $\mathrm{V}$ atoms [8.9]. In the ladder case, only the half-filled bonding band is taken into account, because no electrons are in the antibonding band [8]. The quarter-filled ladder can be mapped on to the half-filled chain by taking only the half-filled bonding band with the empty antibonding band neglected and by an appropriate modification of the parameters $t$ and $J$ [8.17]. In addition, the $t-J$ model has the advantage over a more realistic Hubbard model in that the former can treat larger clusters, which is crucial for the discussion of finite temperature effects. We have calculated $A(k, \omega)$;

$$
A(k, \omega)=\frac{1}{Z} \sum_{f, \sigma} e^{-\beta E_{i}^{N}}\left|\left\langle f, N-1\left|c_{k \sigma}\right| i, N\right\rangle\right|^{2}
$$

$$
\times \delta\left(\omega-E_{i}^{N}+E_{f}^{N-1}\right),
$$

where $Z=\sum_{i} e^{-\beta E_{i}^{N}}$ is the partition function. Results for a half-filled 14-site $t$ - $J$ cluster with $J / t=1 / 3$ at $T=0, J / 4$ and $J / 2$ are shown in Fig. 3 [18]. $A(k, \omega)$ at $T=0$ can be intuitively interpreted as a convolution of spinon and holon excitations, whose dispersional widths are $\sim J$ and $\sim 2 t$, respectively. In this scenario, in the ground state, the holon band is empty while the spinon band is half-filled up to the Fermi momentum $k=\pi / 2$ [2]. The lineshape of the "spinon branch" (see Fig. 3) is determined by the band edge singularity of the holon band whereas that of the "holon branch" is determined by the Fermi edge singularity of the spinon band 19]. At finite temperatures, the spectral weight of the spinon branch at $\omega / t>1(\omega / t<-2)$ is transferred from $0<k<\pi / 2$ $(\pi / 2<k<\pi)$ to $\pi / 2<k<\pi(0<k<\pi / 2)$. At the same time, the intensity of the holon branch decreases. In fact, spectral weight is transferred from the spinon branch to a wide energy region, making the spectral features less pronounced and more symmetric with respect to $k=\pi / 2$. In fact, the singularity of the holon branch due to the existence of the spinon Fermi surface is easily smeared out over the entire energy range of $4 t(\gg T \sim J)$ at finite temperatures of order $J$ [4].

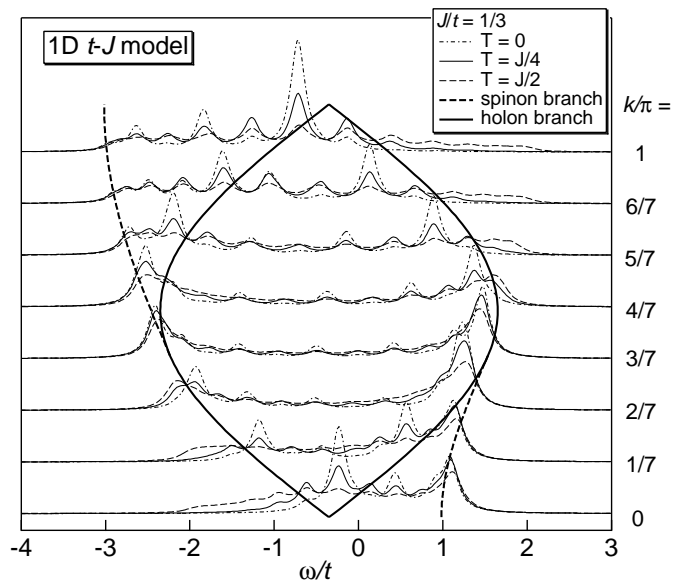

FIG. 3. Spectral function of the half-filled 14-site $t$ - $J$ model with $J / t=1 / 3$ at various temperatures. The thick solid and dashed curves indicate the singularities of the spinon and holon branches, respectively.

In Fig. 2 we show a comparison between the experimental and theoretical spectra. Figure 2 (a) shows experimental spectra for selected momentum values and Figs. 2 (b) and (c) show the intensity plot in the $E$ - $k$ plane on the grey scale. Correspondingly, we show in Figs. 2 (d)-(f) the theoretical spectra at $T=J / 4 \sim 150 \mathrm{~K}$ (solid lines) and $T=J / 2 \sim 300 \mathrm{~K}$ (dashed lines) after Gaussian broadening with the width $2.5 t$ and their intensity plots. Here, we have assumed that $t=3 J=0.15 \mathrm{eV}$, which is plausible because $J=4 t^{2} / U \sim 0.05 \mathrm{eV}$ and $U=2-4$ $\mathrm{eV}$ in typical vanadium oxides 20]. Comparing (a)-(c) 
and (d)-(f), overall agreement is satisfactory. In the low temperature region $(T=120 \mathrm{~K}$ or $J / 4)$, the experimental shift of the peak position between $k_{b}=0.0$ and $0.95 \pi$ may be attributed to the existence of the spinon branch, resulting in the asymmetry of the spectra with respect to $k=\pi / 2$. Besides, between $k_{b}=0.32 \pi$ and $0.72 \pi$, there are two dispersing features which may be assigned to the two holon branches as reproduced in the theoretical spectra. These findings are also substantiated in the comparison between (b) and (e). Unlike the 1D cuprates, where the intense $\mathrm{O} 2 p$ structure obscures the higher binding energy part of the holon and spinon branches [2], the whole structure of theoretical $A(k, \omega)$ can be compared with the experimental results of this compound.

In the high temperature region $(T=300 \mathrm{~K}$ or $J / 2)$, both results become broader and more symmetric with respect to $k=\pi / 2$. As a result, the tendency of the experimental spectral weight redistribution due to finite temperature is grossly reproduced by the theory as seen in the intensity plots in Fig. 2. Around $k \sim 0$, agreement is quite excellent. To this extent, the experimental finite temperature effects may be attributed to the existence of the spinon Fermi surface, which theoretically causes the dramatic spectral redistribution over the entire $E$ - $k$ space with changing temperature. To be more precise, however, there exist some discrepancies between theory and experiment. When the temperature is increased from $120 \mathrm{~K}$ to $300 \mathrm{~K}$, the spectra change more dramatically than the theoretical prediction. Furthermore, while the temperature dependence is rather well simulated by theory at $k_{b} \sim 0-0.32 \pi$, at $k_{b} \sim 0.5 \pi$ the feature around $E_{B}=0.7$ $\mathrm{eV}$ in experiment loses much of its spectral weight in going from $120 \mathrm{~K}$ to $300 \mathrm{~K}$, in disagreement with theory. In addition, around $k_{b} \sim \pi$ in going from $120 \mathrm{~K}$ to $300 \mathrm{~K}$, the experimental spectra lose spectral weight at $E_{B}<0.8$ $\mathrm{eV}$ and a longer tail develops on the high binding energy side unlike the theoretical spectra. At low temperatures, where the decay of a photohole is probably dominated by purely electronic mechanisms while other decay channels may become available at higher temperatures. As a candidate, we may consider electron-phonon interaction and possible charge disorder, which may become important at higher temperatures. The difference between the $t$ - $J$ model and the Hubbard model as well as the degeneracy of the $\mathrm{V} 3 d$ orbitals might be another origin for the discrepancy.

In conclusion, we have made an ARPES study of $\mathrm{Na}_{0.96} \mathrm{~V}_{2} \mathrm{O}_{5}$ by changing the temperature and found that a strong spectral weight redistribution occurs in the lower Hubbard band. Also we have calculated the one-particle spectral function of the $1 \mathrm{D} t-J$ model at finite temperatures by the exact diagonalization method. The overall agreement between theory and experiment implies that the spin-charge separation picture is valid in this system. Although they are more drastic than the theoretical prediction, the experimental finite temperature effects have been partly explained by the theory, which may be expressed as the "Fermi surface" effect of the spinon band.

We would like to thank H. Suzuura, H. Shiba, K. Penc, C. Kim, N. Kawakami, T. Mutou, and D. van der Marel for informative discussions. This work was supported by a Special Coordination Fund from the Science and Technology Agency of Japan. One of us (KK) is supported by a Research Fellowship of the Japan Society for the Promotion of Science for Young Scientists.

[1] E. Lieb and F. Y. Wu, Phys. Rev. Lett., 20, 1445 (1968).

[2] C. Kim,et al., Phys. Rev. Lett. 77, 4054 (1996); Phys. Rev. B 56, 15589 (1998).

[3] K. Kobayashi et al., Phys. Rev. Lett. 80, 3121 (1998).

[4] K. Penc and M. Serhan, Phys. Rev. B 56, 6555 (1997).

[5] M. Isobe and Y. Ueda, J. Alloys and Compounds, 262263, 180 (1997).

[6] A. Carpy and J. Galy, Acta Cryst. B 311481 (1975).

[7] M. Isobe and Y. Ueda, J. Phys. Soc. Jpn. 65, 1178 (1996).

[8] H. Smolinski et al., Phys. Rev. Lett. 80, 5164 (1998).

[9] T. Ohama, H. Yasuoka, M. Isobe, and Y. Ueda, preprint.

[10] It is reasonable because the $4 \%$ deficiency in $\mathrm{Na}$ would have negligible effect on the spin correlation because of the short correlation length $\left(\xi_{A F}\right)$ above $T \sim T_{S P}[\mathrm{H}$. Tsunetsugu, J. Phys. Soc. Jpn. 60, 1460 (1991)].

[11] M.Isobe, C. Kagami and Y.Ueda, J. Crystal Growth, 181, 314 (1997).

[12] T. Ohama et al., J. Phys. Soc. Jpn. 66, 3008 (1997).

[13] S. Hüfner, Photoelectron Spectroscopy (Springer-Verlag, Berlin, 1994).

[14] The origin of the weak but finite intensity for the normal emission, which shows no temperature dependence, is not clear at this moment, as discussed in Ref. [3]. Alternatively, this might be attributed to a background as suggested by C. Kim et al. [2]

[15] We have obtained spectra in Fig. 3 of Ref. [3] by normalizing them to their peak height rather than the area. It is noted that the spectra of the more insulating $\mathrm{Na}_{1.00} \mathrm{~V}_{2} \mathrm{O}_{5}$ spectra were slightly distorted toward higher $E_{B}$ due to weak charging effect even at $300 \mathrm{~K}$.

[16] T. Tohyama, H. Okuda, and S. Maekawa, Physica C 215, 382 (1993).

[17] P. Horsch and F. Mack, Eur. Phys. J. B 5, 367-370 (1998).

[18] Na vacancies are more sparsely distributed than the cluster size used for the exact diagonalization calculation, which justifies our quantitative comparison hereafter.

[19] H. Suzuura and N. Nagaosa, Phys. Rev. B 56, 3548 (1997); S. Sorella and A. Parola, J. Phys. Cond. Matter 4, 3589 (1992).

[20] K. Morikawa et al., Phys. Rev. B 52, 13711 (1995); S. Shin et al., Phys. Rev. B 41, 4993 (1990); A.E. Bocquet et al., Phys. Rev. B 53, 1161 (1996). 